\title{
Comparison of the effects and side-effects of sedation with propofol versus midazolam plus pethidine in patients undergoing endoscopy in Imam Khomeini Hospital, Ahvaz
}

\author{
Eskandar Hajiani ${ }^{1}$, Jalal Hashemi ${ }^{1}$, Jalal Sayyah ${ }^{2}$ \\ ${ }^{1}$ Research Institute for Infectious Diseases of Digestive System and School of Medicine, Ahvaz Jundishapur University \\ of Medical Sciences, Ahvaz, Iran \\ ${ }^{2}$ Student Research Committee, Ahvaz Jundishapur University of Medical Sciences, Ahvaz, Iran
}

Gastroenterology Rev 2018; 13 (3): 228-233

DOI: https://doi.org/10.5114/pg.2018.78288

Key words: endoscopy, propofol, midazolam, pethidine.

Address for correspondence: Jalal Sayyah, Student Research Committee, Ahvaz Jundishapur University of Medical Sciences, 123456 Ahvaz, Iran, phone: +98 9163117541, e-mail: sayyahjalal@gmail.com

\begin{abstract}
Introduction: Gastrointestinal endoscopy is an invasive and diagnostic procedure that causes the patients considerable pain, discomfort, and anxiety. Therefore, various types of sedation and analgesia techniques have been used during the procedure.

Aim: To compare the effects and side-effects of sedation with propofol versus midazolam plus pethidine in patients undergoing endoscopy.

Material and methods: This is a randomised controlled double-blind clinical trial study conducted on 272 patients undergoing diagnostic and treatment endoscopy and colonoscopy in Imam Khomeini Hospital in Ahvaz between 2017 and 2018. The patients were randomly assigned to two groups. Patients in the first group $(n=136)$ received propofol with midazolam and ketamine, and the second group $(n=136)$ received pethidine and midazolam. Study outcome measures included the recovery time, patient satisfaction, quality of sedation, and adverse events.

Results: The occurrence of complications was higher in the propofol group ( $25 \%$ vs. $0 \% ; p=0.0001)$. No serious adverse events were observed in the study groups. Overall patient satisfaction and quality of sedation assessment scores in the propofol group were significantly better than those seen in the pethidine-midazolam group ( $p=0.012$ and $p=0.001$, respectively). Recovery time was statistically shorter in the propofol-midazolam group $(6.05 \pm 1.62 \mathrm{~min})$ compared to the pethidine-midazolam group $(6.72 \pm 2.21 \mathrm{~min})(p=0.006)$.

Conclusions: Propofol-midazolam can provide better sedation, patient satisfaction, and recovery than pethidine-midazolam during endoscopy. Therefore, it can be recommended in patients scheduled for diagnostic and treatment endoscopy.
\end{abstract}

\section{Introduction}

Endoscopy and colonoscopy are relatively safe medical interventions frequently used worldwide due to their intrinsic properties and diagnostic capabilities. However, most patients are afraid of endoscopy and colonoscopy, and it is uncomfortable and painful for them, which makes it difficult for doctors to perform such actions $[1,2]$. Thereby, application of sedation protocols by intravenous injections is used to calm and relieve pain in most developed countries. Sedation is the most effective strategy for endoscopy, and most patients prefer to undergo endoscopy by sedation.
The initial purpose of the sedation process is to reduce the anxiety and discomfort of the patient, thereby improving the tolerance and satisfaction of the endoscopic process [3]. Sedation also reduces the risk of physical damage to the patient during the examination and provides ideal conditions for complete examination and better diagnosis with lower error rates for endoscopy $[4,5]$. However, sedation may result in delay of recovery and clearance of the patient and also increases the cost of endoscopy and risk of cardiovascular complications $[3,6]$. Despite the above explanations, application of sedation in the endoscopic process is increasing worldwide 
[6]. The American Society of Anaesthesiologists (ASA) and many others have reported that endoscopic sedation could be done by people other than an anaesthetist (gastroenterologists and endoscopists) [7-10].

Until recently, treadmill sedations were implemented routinely in endoscopy using a combination of benzodiazepines, such as midazolam. The combination of these factors was used to achieve a conscious sedation. This method takes a long time for the drug to be effective [6]. Today, the use of a propofol-based sedation is increasing $[11,12]$. Propofol is a short-acting hypnotist agent that, according to gastroenterologists, is preferable to many older drugs due to its fast action, good patient and doctor satisfaction, and very short recovery time [7, 13].

Because propofol has no antinociceptive effect, application of propofol alone is not effective for a moderate level of sedation in endoscopy. So, in endoscopy, propofol is prescribed together with an opioid and/or benzodiazepines, even at low doses [14]. The use of propofol-oopyhydes combinations for the creation of a moderate sedation level increases the safety of the propofol profile by reducing the need for deep sedation and general anaesthetic [6].

Given the increasing need for endoscopic and colonoscopic diagnostic methods and the necessity of the application of sedation agents for patient comfort, also facilitating the physicians' actions during this operation, it seems to be necessary to know the extent and frequency of adverse effects of sedation in patients.

\section{Aim}

Because quantitative studies have compared the efficacy and side effects of sedation drugs such as propofol in endoscopy, the aim of this study was to evaluate the efficacy and side effects of sedation with propofol protocol, midazolam protocols, and pethidine in endoscopic patients in Ahvaz Imam Khomeini Hospital.

\section{Material and methods}

The present study is a double-blind, randomised clinical trial that was performed on patients referred to endoscopy and diagnostic and treatment colonoscopy in Ahwaz Imam Khomeini Hospital during the second half of the year 2017. After obtaining permission from Ahvaz Jundishapur University of Medical Sciences Ethics Committee and giving explanations by the researchers to the patients about the purpose of and how to perform the plan, they entered the research case study by obtaining informed and written consent. In this study, 272 patients over the age of 18 years with ASA class I-II (American society of anaesthesiologists) underwent endoscopic sedation. Patients with unstable vital con- ditions signs such as fever, tachycardia, systolic blood pressure lower than $90 \mathrm{~mm} \mathrm{Hg}$, tachypnoea, allergy to anaesthetic drugs, and patients with metabolic, pulmonary, neurological, and psychological disorders, were excluded from the study.

\section{Taking intervention}

After initial evaluation of patients (taking history and complete examination) and recording the results in the case, the patients were randomly assigned into two groups and anaesthetised. The drug was injected to the patients one minute before starting endoscopy. The first group (136 patients) underwent sedation with propofol protocol (propofol $20 \mathrm{mg}+$ midazolam $1 \mathrm{mg}$ + ketamine $20 \mathrm{mg}$ ), while the second group (136 patients) underwent sedation with midazolam $(2.5 \mathrm{mg})$ and pethidine (25 mg). After endoscopy, the patients were transferred to the recovery room and monitored for any side effects throughout the entire period.

In the case of any side effect, diagnostic and therapeutic facilities including complete blood count (CBC) tests, blood urea nitrogen (BUN), creatinine, and pulse oximetry tests were performed and, if necessary, hospitalisation possibility was provided by the researcher.

In this study, the drugs were randomly prescribed to patients to ensure compliance with the double-blind protocol. Patients and result evaluation individuals were not informed about the type of anaesthetic trial, and hence this study remained double-blind until the end.

\section{Patient evaluation}

Before the treatment, demographic data of each patient (including age and gender) and clinical information including the presence of underlying illness and other vital parameters were collected by referring to the file and recorded in the form of the patient's questionnaire. Also, the recovery time (from discontinuation of infusion to reaching Aldrete criteria 9 and higher), side effects of sedation (hypoxia, nausea, vomiting, hypotension, respiratory, and cardiac problems, etc.) during endoscopy, sedentary level of consciousness and patients' satisfaction were reviewed and recorded. The proper sedentary level of consciousness was studied in three scales: 1) a completely calm patient; 2 ) reliable tolerant patient; and 3) completely restless patient. Also, satisfaction level and comfort of the patient through endoscopy was measured as good, medium, or bad.

\section{Statistical analysis}

SPSS software version 22 was used for statistical analysis. Data were analysed by descriptive statistics including frequency, mean, standard deviation, frequency, and frequency percentage. $T$-test was used for evaluat- 
Table I. Patients' characteristics

\begin{tabular}{lcccc} 
Group & Number & Age & Male gender & Upper location \\
\hline Midazolam + pethidine & 136 & $46.87 \pm 16.25$ & $70(47.51 \%)$ & $65(80.47 \%)$ \\
\hline Midazolam + propofol & 136 & $52.78 \pm 18.84$ & $86(23.63 \%)$ & $95(85.69 \%)$ \\
\hline -value & & 0.006 & 0.066 & 0.0001
\end{tabular}

Table II. Comparison of the efficacy of the two protocols

\begin{tabular}{|c|c|c|c|c|c|c|c|}
\hline \multirow[t]{2}{*}{ Group } & \multirow[t]{2}{*}{ Side effects } & \multirow[t]{2}{*}{ Recovery time } & \multicolumn{2}{|c|}{ Level of sedation } & \multicolumn{3}{|c|}{ Satisfaction } \\
\hline & & & Complete & Relatively & Good & Middle & Bad \\
\hline $\begin{array}{l}\text { Midazolam + } \\
\text { pethidine }\end{array}$ & 0 & $6.72 \pm 2.21$ & $67(26.49 \%)$ & $69(74.50 \%)$ & $90(18.66 \%)$ & $45(33.08 \%)$ & $1(74.0 \%)$ \\
\hline $\begin{array}{l}\text { Midazolam + } \\
\text { propofol }\end{array}$ & $34(25 \%)$ & $6.05 \pm 1.62$ & $103(74.75 \%)$ & $33(26.24 \%)$ & 111 (62.81\%) & $25(38.18 \%)$ & 0 \\
\hline$P$-value & 0.0001 & 0.006 & \multicolumn{2}{|c|}{0.0001} & & 0.012 & \\
\hline
\end{tabular}

ing the level of significance of variations and comparing the quantitative variables, and Mann-Whitney test was applied to compare quantitative variables with abnormal distribution. Chi square $\left(\chi^{2}\right)$ test was used to analyse the qualitative variables. The level of significance in the tests was considered to be 0.05 .

\section{Results}

A total of 156 (57.35\%) males and 116 (42.65\%) females participated in the present study (Table I). The

Table III. Relationship between the incidences of side effects with the variables

\begin{tabular}{|c|c|c|c|}
\hline Variable & $\begin{array}{l}\text { With side } \\
\text { effects }\end{array}$ & $\begin{array}{l}\text { Without side } \\
\text { effects }\end{array}$ & $P$-value \\
\hline Age & $60.82 \pm 21.60$ & $50.07 \pm 17.09$ & 0.011 \\
\hline \multicolumn{4}{|l|}{ Gender: } \\
\hline Male & $23(65.67 \%)$ & $63(76.61 \%)$ & \multirow[t]{2}{*}{0.682} \\
\hline Female & 11 (35.32\%) & 39 (24.38\%) & \\
\hline Recovery time & $6.50 \pm 1.95$ & $5.90 \pm 1.47$ & 0.063 \\
\hline \multicolumn{4}{|c|}{ Endoscopy area: } \\
\hline Upper & $22(71.64 \%)$ & 73 (57.71\%) & \multirow[t]{2}{*}{0.519} \\
\hline Lower & $12(29.53 \%)$ & 29 (43.28\%) & \\
\hline \multicolumn{4}{|c|}{ Level of sedation: } \\
\hline Complete & $28(35.82 \%)$ & 75 (53.73\%) & \multirow[t]{2}{*}{0.361} \\
\hline Relatively & $6(65.17 \%)$ & 27 (47.29\%) & \\
\hline \multicolumn{4}{|l|}{ Satisfaction: } \\
\hline Good & 29 (29.85\%) & 82 (39.80\%) & \multirow[t]{2}{*}{0.616} \\
\hline Middle & 5 (71.14\%) & 20 (61.19\%) & \\
\hline
\end{tabular}

results of statistical analysis did not show any significant difference between the genders of the individuals in these two groups $(p=0.66)$. The mean age of the patients under study in the pethidine + midazolam group was $46.87 \pm 16.26$ years old (range: $19-85$ years), while in the group undergo sedation with propofol protocol, this value was $52.88 \pm 18.84$ years old (range: $18-92$ years). There was a significant difference in the age of individuals of the two groups $(p=0.006)$.

The results of the efficacy of the two protocols (Table II) showed that the mean recovery time in patients sedated with the pethidine + midazolam protocol, which was $6.72 \pm 2.21$ (range: 12-15) min, was significantly higher than that of the propofol protocol $6.05 \pm 1.62$ (range: $3-10 \mathrm{~min}),(p=0.006)$. Also, the level of sedation and satisfaction of patients sedated with propofol protocol was higher than that of the pethidine+midazolam protocol ( $p=0.001$ and $p=0.122$, respectively).

In the present study, no serious side effects were observed in either of the two groups. Also, the patients who underwent the sedation with pethidine + midazolam had no side effects. However, 34 (25\%) of the patients who underwent sedation with propofol protocol showed side effects. Therefore, there was a significant difference in the observed side effects of the two groups $(p<0.001)$. Side effects included 15 cases of hallucinations, 9 cases of hypoxia, and 10 cases of hypotension and bradycardia. No significant differences were observed in the side effects observed in patients subjected to upper and lower endoscopies ( $p=0.193$ ).

In addition, as shown in Table III, there was no significant relationship between the presence of side effects and gender, recovery time, endoscopic location, sedation, and patient satisfaction from endoscopy 
$(p>0.05)$. However, the mean age of patients with side effects was significantly higher than those with no side effects $(p=0.1111)$.

In both of the examined sedation protocols, endoscopic patients had more sedation and satisfaction than colonoscopy patients $(p<0.05)$. However, there was no significant difference in the age and gender of the patients and the side effects in endoscopy and colonoscopy in any of the sedation protocols $(p>0.05)$. The lower recovery time was identical in propofol protocols in patients of endoscopy and colonoscopy, but in the pethidine-midazolam sedation protocol, recovery time of endoscopic patients was lower than colonoscopy patients.

\section{Discussions}

The present study evaluated the efficacy and safety of two protocols including propofol-midazolam and petidine-midazolam in endoscopy and colonoscopy diagnostic and therapeutic. The results of the upper level showed more comfort and satisfaction of patients and a shorter recovery time in the propofol-midazolam group. These results are in agreement with the findings of previous studies, in which it was stated that propofol in combination with other sedatives is an appropriate option for achieving sedation with proper depth, and high tolerance for the patient, and is free of the side effects associated with injection of high doses of propofol, such as increasing the recovery time [8, 15-17].

In the present study, no serious side effect caused by sedation was observed in the two groups. In this study, patients who underwent sedation with the pethidine + midazolam protocol showed no side effects, while $25 \%$ of patients who underwent sedation with propofol showed a slight level of side effects (including hallucinations, hypoxia, hypotension, and bradycardia), so these haemodynamic changes and respiratory complications were temporary and did not require any special intervention. Given these side effects, especially in people with underlying cardiovascular disease, further investigation is required. Also, the observation of more side effects in the propofol group could be related to the fact that these patients have a higher mean age than in the peptide-midazolam group.

Studies have shown that, despite the effectiveness of sedation by propofol, there is still a risk of cardio respiratory side effects such as hypoxia, hypotension, arrhythmias, and respiratory distress in the case of use of propofol, which requires cardiopulmonary support [18]. In the study of Alatise et al. [17] hypotension was similar in the three protocols of sedation by propofol-midazolam, opioid-midazolam, and propofol alone, but the cause of this similarity was unknown. However, the emergence of hypoxaemia in a group with propofol (100\% of patients in the group of sedation with propofol and $97.5 \%$ of patients in the propofol-midazolam group) was significantly higher than in the opioid-midazolam (30\%) group.

Because in the study of Alatise et al. [17] hypoxaemia was observed in a significant number of patients under opioid-midazolam sedation, they emphasised that careful monitoring of patients is vital without considering the sedation. In the study of Lera dos Santos et al. [8] there were no serious side effects in both groups of sedation: with propofol-fentanyl and midazolam-fentanyl. However, temporary and mild hypoxia was seen in $42 \%$ of patients in the propofol group and $26 \%$ of patients in the midazolam group, and the two groups showed a significant difference in this case. Temporal hypotension was seen in $11 \%$ of the midazolam group and $26 \%$ of the patients in the propofol group. The possibility of side effects in their study is greater than the present study. Also, in the study of Amornyotin et al. in Thailand, no serious side effects were observed in the two sedation protocols of propofol-pethidine and midazolam-fentanyl, but the extent of temporal and minor side effects caused by sedation in propofol-pethidine protocol (including hypoxaemia and hypotension) was higher [19].

On the other hand, in the study of Paspatis et al. [16] no serious side effects caused by sedation were observed, but the results of the temporary cardiopulmonary bypasses were similar in both groups of propofol-midazolam and midazolam-pethidine. In the study of Mohammad Alizadeh et al. [20], the incidence of complications of sedation in the midazolam-fentanyl and propofol-fentanyl groups were $40 \%$ and $20 \%$, respectively (including nausea, hypotension, and hypoxaemia), but there was no significant difference between the two groups. In another study conducted by Pascual et al. in Cuba, it was reported that the results of application of two sedation protocol including propofol and midazolam + pethidine in colonoscopy were identical in terms of side effects [21]. Also, in another study, a comparison of the sedation with midazolam-propofol and midazolam-meperidone in 150 high-risk patients (ASA grade III and higher) showed that the propofol group did not have greater side effects than the other group [12]. These results are not consistent with the findings of this study. This could be attributed to the significant difference in the mean age values of the two groups in the present study.

In the present study, there was no significant relationship between the presence of side effects and gender, recovery time, endoscopy (upper/lower), sedation, and satisfaction. But the mean age of patients with 
complications was significantly higher than uncomplicated patients. Previous studies also showed that the age of patients was an independent risk factor for significant reduction in $\mathrm{O}_{2}$ saturation [16]. However, the study conducted by Alatise et al. [17] did not confirm this. Nonetheless, it is recommended that the intranasal oxygen and blood pressure be monitored during the endoscopy and colonoscopy regardless of the used sedation regime.

Differences in the incidence of side effects in various studies could be also attributed to differences in the population and the study samples and characteristics of patients, including age, respiratory diseases, and other factors such as the dose of the drug. Therefore, conduction of further studies with greater sample size in a multi-centred approach is necessary to confirm these results.

The results of the present study showed that the recovery time in the group under sedation with propofol protocol was significantly lower than that of pethidine + midazolam. In a study conducted by Paspatis et al. [16] it was shown that due to the shorter recovery time a lower dosage of midazolam in combination with propofol was more effective than the combination of midazolam and opioid pethidine during colonoscopy. In the study conducted by Hosseini et al. [22], recovery time in the propofol-fentanyl group was significantly lower than that of the meperidine-midazolam group. In the study of Alatise et al., the recovery time in patients who underwent diagnostic colonoscopy in the propofol-midazolam group (5.8 min) was significantly lower than the that of the opioid-midazolam group (13.4 min) and propofol alone (7.9 min) [17]. In another study, Padmanabhan et al. [23] showed that endoscopy could be carried out using a combination of low-dosage propofol with a narcotic and/or benzodiazepine agent at moderate sedation levels, and a combination of small doses of propofol with a narcotic agent and midazolam does not prolong the patients' recovery time. The results of the study performed by Lera dos Santos et al. [8] in Italy showed that the recovery and clearance time in the propofol-fentanyl group was significantly lower than that of endoscopy with midazolam-fentanyl sedation. Similarly, Vargo et al. [24] as well as Koshy et al. [25] reported that prescribing propofol significantly reduced the recovery time after endoscopy compared to the combination of midazolam and meperidine. These results are consistent with the findings of this study.

In the present study, the sedentary level of consciousness and satisfaction of patients in sedation with the propofol protocol was significantly higher than that of the pethidine + midazolam protocol. The results of the study conducted by Hosseini et al. [22] showed that the level of sedation and satisfaction of patients who underwent colonoscopy in the group sedated with meperidine-midazolam was significantly lower than that of the propofol-fentanyl group. The results of the study carried out by Paspatis et al. [16] in Greece showed that the patient's comfort level in colonoscopy with midazolam-pethidine sedation was significantly higher than the standard regime of midazolam-propofol protocol. In the study of Alatise et al. [17], satisfaction of the physician and patient from propofol-based sedation was significantly higher than opioid-midazolam sedation. In the study of Amornyotin et al. conducted in Thailand, it was reported that patients' and physician's satisfaction during endoscopy of patients who underwent deep sedation with propofol and pethidine was significantly higher than that of midazolam and fentanyl [19]. The results of the study conducted by Agostoni et al. in Italy showed that the protocol of sedation with propofol-opioid (fentanyl) is a safe and effective method for endoscopy of the upper gastrointestinal endoscopy because the patients and endoscopists have high levels of satisfaction [15]. These results are consistent with the findings of this study.

The present study also encountered some limitations, including: failure to check the level of sedation (OAA/S and BIS), clearance time and endoscopy duration, and the lack of evaluation of preoperative anxiety, which has been reported as a factor in successful completion of endoscopic process. Also, the randomisation of two treatment groups was not performed well in terms of age.

\section{Conclusions}

The results of this study showed that recovery time in the propofol group was significantly lower and the level of satisfaction and relaxation was higher. Therefore, prescription of the propofol-midazolam combination by endoscopists is more effective than pethidine-midazolam. Hence, regarding the beneficial effects of propofol-midazolam in sedation and shortening of recovery time, and creating more satisfaction levels in patients, application of this compound in treatment of patients undergoing endoscopy is recommended.

\section{Conflict of interest}

The authors declare no conflict of interest.

\section{References}

1. Amornyotin S. Sedation and monitoring for gastrointestinal endoscopy. World J Gastrointest Endosc 2013; 5: 47-55.

2. Triantafillidis JK, Merikas E, Nikolakis D, Papalois AE. Sedation in gastrointestinal endoscopy: current issues. World J Gastroenterol 2013; 19: 463-81. 
3. Riphaus A, Rabofski M, Wehrmann T. Endoscopic sedation and monitoring practice in Germany: results from the first nationwide survey. Z Gastroenterol 2010; 48: 392-7.

4. American Society for Gastrointestinal Endoscopy. Multisociety Sedation Curriculum for Gastrointestinal Endoscopy. Hepatology 2012; 56: E1-25.

5. Baudet JS, Borque P, Borja E, et al. Use of sedation in gastrointestinal endoscopy: a nationwide survey in Spain. Eur J Gas troenterol Hepatol 2009; 21: 882-8.

6. Dumonceau JM, Riphaus A, Aparicio JR, et al. European Society of Gastrointestinal Endoscopy, European Society of Gastroenterology and Endoscopy Nurses and Associates, and the European Society of Anaesthesiology Guideline: non-anesthesiologist administration of propofol for Gl endoscopy. Endoscopy 2010; 42: 960-74.

7. Cohen LB, Wecsler JS, Gaetano JN, et al. Endoscopic sedation in the United States: results from a nationwide survey. Am J Gastroenterol 2006; 101: 967-74.

8. Lera dos Santos ME, Maluf-Filho F, Chaves DM, et al. Deep sedation during gastrointestinal endoscopy: propofol-fentanyl and midazolam-fentanyl regimens. World J Gastroenterol 2013; 19: 3439-46.

9. Practice guidelines for sedation and analgesia by non-anesthesiologists. Anesthesiology 2002; 96: 1004-17.

10. Sedation and anesthesia in Gl endoscopy. Gastrointest Endosc 2008; 68: 815-26.

11. Rex D, Deenadayalu V, Eid E, et al. Endoscopist-directed admin istration of propofol: a worldwide safety experience. Gastroenterology 2009; 137: 1229-37.

12. Riphaus A, Stergiou N, Wehrmann T. Sedation with propofol for routine ERCP in high-risk octogenarians: a randomized, controlled study. Am J Gastroenterol 2005; 100: 1957-63.

13. Brill JV. Endoscopic sedation: legislative update and implications for reimbursement. Gastrointest Endosc Clin N Am 2008; 18: 665-78.

14. Paspatis G, Manolaraki M, Tribonias G, et al. Endoscopic sedation in Greece: results from a nationwide survey for the Hellenic Foundation of gastroenterology and nutrition. Dig Liv Dis 2009; 41: 807-11.

15. Agostoni M, Fanti L, Arcidiacono PG, et al. Midazolam and pethidine versus propofol and fentanyl patient controlled sedation/analgesia for upper gastrointestinal tract ultrasound endoscopy: a prospective randomized controlled trial. Dig Liver Dis 2007; 39: 1024-9.

16. Paspatis GA, Manolaraki M, Xirouchakis G, et al. Synergistic sedation with midazolam and propofol versus midazolam and pethidine in colonoscopies: a prospective, randomized study. Am J Gastroenterol 2002; 97: 1963-7.

17. Alatise OI, Owojuyigbe AM, Yakubu MA, et al. Propofol versus traditional sedative methods for colonoscopy in a low-resource setting. Niger Postgrad Med J 2015; 22: 151-7.

18. Coté GA, Hovis RM, Ansstas MA, et al. Incidence of sedation-related complications with propofol use during advanced endoscopic procedures. Clin Gastroenterol Hepatol 2010; 8 137-42.

19. Amornyotin S, Songarj P, Kongphlay S. Deep sedation with propofol and pethidine versus moderate sedation with midazolam and fentanyl in colonoscopic procedure. J Gastroenterol Hepatol Res 2013; 2: 885-90.
20. Mohammad Alizadeh AH, Farhanchi A, Falah M, et al. Comparative evaluation of conscious sedation effect of propofol versus midazolam in endoscopic and colonoscopic procedures. J Zanjan Univ Med Sci Health Serv 2002; 10: 29-33.

21. Pascual MG, Zayas Berbes M, Sáez Baños M, et al. Propofol versus midazolam and pethidine in the colonoscopy realization. Acta Gastroenterol Latinoam 2011; 41: 214-20.

22. Hosseini V, Fakheri H, Zamani A, et al. Comparison of meperidine-midazolam and propofol-fentanil sedation in patients scheduled for elective colonoscopy. J Mazand Univ Med Sci 2014; 23: 17-22.

23. Padmanabhan U, Leslie K. Australian anaesthetists' practice of sedation for gastrointestinal endoscopy in adult patients. Anaesth Intensive Care 2008; 36: 436-41.

24. Vargo JJ, Zuccaro G Jr, Dumot JA, et al. Gastroenterologist-administered propofol versus meperidine and midazolam for advanced upper endoscopy: a prospective, randomized trial. Gastroenterology 2002; 123: 8-16.

25. Koshy G, Nair S, Norkus EP, et al. Propofol versus midazolam and meperidine for conscious sedation in GI endoscopy. Am J Gastroenterol 2000; 95: 1476-9.

Received: 9.01.2018

Accepted: 11.04.2018 